\title{
Concurrent anti-stress urinary incontinence (SUI) surgery for advanced pelvic organ prolapse (POP) surgery and SUI: an Asian perspective footnote from the Pan-Asia meeting
}

\author{
Tsia-Shu Lo ${ }^{1,2,3,4,5} \cdot$ Yiap Loong Tan ${ }^{6}$ \\ Received: 29 April 2021 / Accepted: 19 May 2021 / Published online: 31 May 2021 \\ (C) The International Urogynecological Association 2021
}

There are many conflicting opinions regarding concurrent anti-incontinence surgery performed at the time of surgery to correct pelvic organ prolapse (POP). In the setting of overt concomitment stress urinary incontinence (SUI), most investigators would perform an anti-incontinence procedure but the question of occult SUI is more vexing.

To better understand how investigators from Asian countries view this complex issue, we surveyed Asian participants during the International Urogynaecology Association (IUGA) meeting in Vienna, 2018. We were interested to find out what the practice was for preforming a concurrent mid-urethral sling with POP surgery for women who have occult or overt SUI. In addition, we were interested in the management of mixed urinary incontinence (MUI) (predominantly SUI) with advanced POP surgery.

Participants from 13 countries responded. Representatives from five countries reported performing anti-SUI surgery >

Tsia-Shu Lo

2378@cgmh.org.tw

1 Division of Urogynecology, Department of Obstetrics and Gynecology, Linkou, Chang Gung Memorial Hospital, Linkou Medical Center, 5, Fu-Hsin Street, Kwei-shan, Tao-Yuan City, Taiwan 333, Republic of China

2 Department of Obstetrics and Gynecology, Chang Gung Memorial Hospital, Keelung Medical Center, Keelung, Taiwan, Republic of China

3 Department of Obstetrics and Gynecology, Chang Gung Memorial Hospital, Taipei Medical Center, Taipei, Taiwan, Republic of China

4 School of Medicine, Chang Gung University, Taoyuan, Taiwan, Republic of China

5 The Asia International Advisory Board (IAB) Representative (20152018), International Urogynecology Association (IUGA), Burnsville, MN, USA

6 Kuching Specialist Hospital, KPJ Healthcare Group, Kuching, Sarawak, Malaysia
$50 \%$ of the time in conjunction with POP surgery, in most cases due to the presence of overt SUI (Fig. 1). The two countries least likely to perform concurrent anti-SUI surgery are Japan and Thailand. About one in every four women who undergo POP surgery in China, Hong Kong and India will have concurrent anti-SUI surgery, in the majority ( $\sim 90 \%)$ only in the setting of concomitant overt SUI.

Of the 13 countries, most surgeons (10 countries) responded that they would perform concurrent anti-SUI surgery only in the presence of overt SUI, while investigators from 3 countries (Malaysia, Philippines and Taiwan) responded that anti-incontinence surgery would be performed in the setting of both overt and occult SUI. There is a marked difference between practices around the Asian continent, and these practices are not well reflected by advanced training or the level of expertise among investigators.

In the setting of mixed urinary incontinence (MUI) and POP, surgeons from Philippines, Singapore, India, Pakistan, Thailand and Taiwan would offer anti-incontinence surgery at the same time as POP surgery (Fig. 2). The rest of the respondents would only offer anti-incontinence surgery in select cases.

The rate of concurrent anti-incontinence and POP surgery appears high in most Asian countries, but there are still some respondents who prefer a two-stage procedure, first correcting the POP. Their reasoning is to reduce surgical complications, postoperative voiding dysfunction or a negative impact on surgical success in treating the POP. Since surgical restoration of the anatomical defects of POP alone provides improvement in MUI symptoms around $50 \%$ of the time, this could be one of the many reason(s) for a two-stage approach, correcting the POP first and then performing a second-stage anti-incontinence surgery when indicated.

The variety of opinions in Asian investigators reflects the variety of opinions in the literature and the issues of how to manage occult SUI and MUI in the setting of POP. This is an area that begs for some evidence-based practice guidelines or 
Fig. 1 Concurrent anti-SUI surgery for advanced POP and SUI in 13 selected countries/areas,

Asia, 2018
Concurrent anti-SUI surgery for advanced POP and SUI

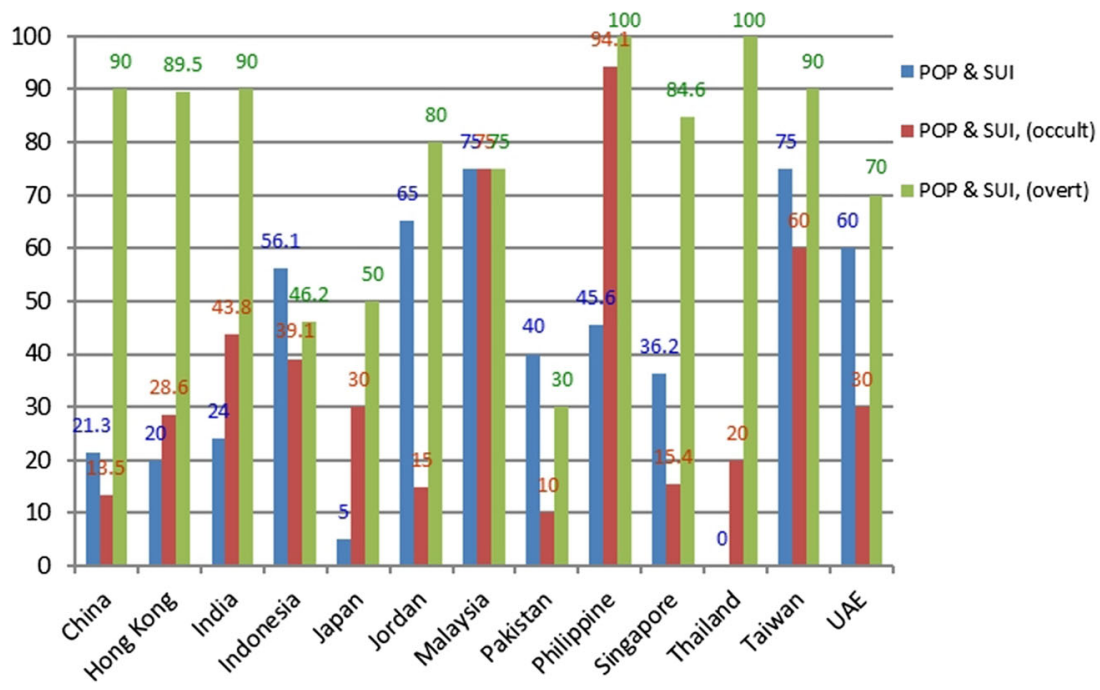

Concurrent anti-SUI surgery for POP and MUI

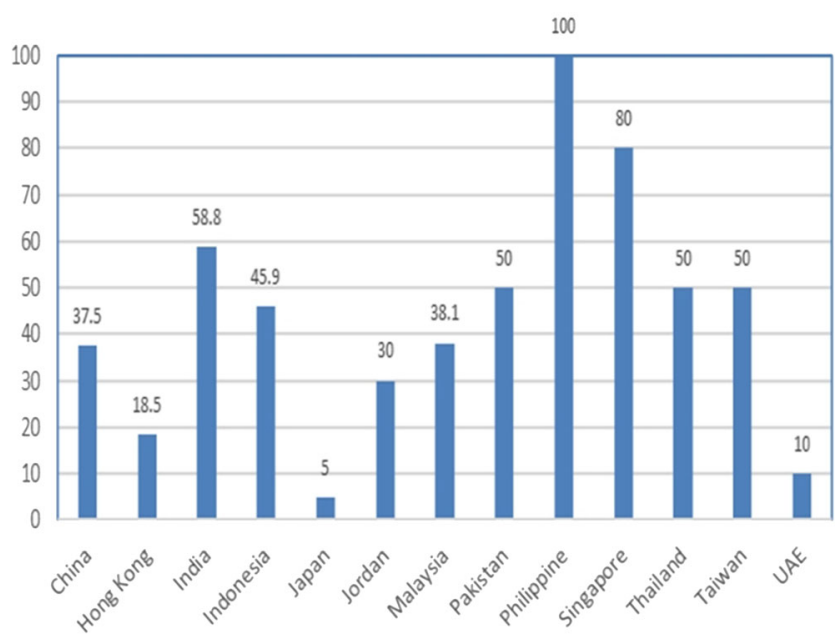

Fig. 2 Concurrent anti-SUI surgery for advanced POP and MUI in 13 selected countries/areas, Asia, 2018 recommendations; otherwise, we will continue to see a wide variety of practice patterns that will be based on local standards of care.

\section{Declarations}

Conflicts of interest None.

Publisher's note Springer Nature remains neutral with regard to jurisdictional claims in published maps and institutional affiliations. 